\title{
A TOOL IN DIFFERENTIAL DIAGNOSIS OF A "CYSTIC" BONE LESION
}

doi: $10.2478 /$ rojost-2018-0043

\author{
A.N. Marinescu, A.I. Nicula, G. lana \\ "Carol Davila" University of Medicine and Pharmacy, Bucharest Romania \\ Department of Medical Imaging, University Emergency Hospital, Bucharest, Romania
}

It is a power point program, which through a succession of steps, allows the obtaining of one shorter diagnosis, from a long list of possible ones, by choosing a character of the bone lesion. The aim of this program is to facilitate the differential diagnosis of this particular aspect of a bone lesion - the cystic, expansile osteolytic bone lesion - from a radiological point of view, but not only. The location, age, pain and other condition will be considered and will represent the first criteria in the differentiation. In this differential diagnosis, we discovered not only benign tumor lesions but also malign bone lesions or other non-tumoral conditions. We considered that this program could be easily used by the radiologist or the orthopedist in training and useful by also employing a representative photo gallery.

Keywords: expansile cystic bone lesion, compression bone atrophy 